\title{
P02-226
}

\section{VENTRAL PREFRONTAL FUNCTION MEDIATES RESILEINCE TO BIPOLAR DISORDER: AN FMRI STUDY OF BD PATIENTS}

AND THEIR UNAFFECTED SIBLINGS

M. Haldane, M. Kempton, S. Frangou

Section of Neurobiology of Psychosis, Institute of Psychiatry, Kings College London, London, UK

Background and aims: Bipolar disorder (BD) is characterised by emotional dysregulation; abnormal emotional information processing is likely to be a component of genetic predisposition to BD.

Method: Functional magnetic resonance imaging data was collected during an event-related facial affect recognition task (fearful, angry, sad expressions), from: 41 BDI patients, 22 of their unaffected siblings, and 51 controls. A random effects analysis was implemented using SPM5.

Results: Patients, relative to controls had significantly:

a. reduced activation in the left inferior frontal gyrus and middle occipital gyrus and,

b. enhanced activation bilaterally in the posterior cingulate and in the left postcentral gyrus; in the temporal lobe, increased activation was seen in the hippocampus and amygdale bilaterally and in the middle and inferior temporal gyri.

Siblings, relative to controls showed significantly enhanced activation in the inferior frontal gyrus and in the parahippocampal gyrus and amydgala. Siblings relative to patients had significantly higher activation in the inferior frontal gyrus.

Conclusions: In BD patients there is evidence of increased limbic activation and decreased cortical efficiency during facial affect processing; increased ventral PFC activation in siblings, in the presence of increased limbic activation, may serve as a compensatory mechanism mediating resilience to disease expression. 